\title{
Remission of Type 2 Diabetes in 8 Hypogonadal Men on Long-Term Testosterone Treatment: Results from a Registry Study
}

KS Haider ${ }^{1}$, A Haider ${ }^{1}$, F Saad 2,3

1Private Urology Practice, Bremerhaven, Germany ${ }^{2}$ Medical Affairs Andrology, Bayer AG, Berlin, Germany ${ }^{3}$ Gulf Medical University School of Medicine, Ajman, UAE

Background

Correcting hypogonadism in men with type 2 diabetes mellitus (T2DM) can be beneficial.

To study long-term effectiveness and safety of testosterone undecanoate injections (TU).

\section{Methods}

Registry of hypogonadal men with testosterone $\leq 12.1 \mathrm{nmol} / \mathrm{L}$ since 2004 in a urological setting. $286 / 776$ patients $(36.9 \%$ ) had type 2 diabetes which had been diagnosed and is treated elsewhere (diabetologist).

133 men received TU $1000 \mathrm{mg} / 12$ weeks (T-group) following an initial 6-week interval. Of these, 8 patients stopped their diabetes medication upon decision by the diabetologist. Thei $\mathrm{HbA}_{1 \mathrm{c}}$ consistently remained below $6.5 \%$. Patient's individual data are reported.

This is the first report suggesting that long-term testosterone treatment in hypogonadal men may result in remission of T2DM.

Conclusion sustainably improved glycaemic control as well as weight to an extent that standard diabetes treatment could be discontinued. Trough total testosterone $(\mathrm{nmol} / \mathrm{L})$ in 133
Th hypogonadal men with T2DM on long-term treatment hypogonadal controls



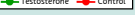

Results

\#1:T2DM since 2000, received metformin since 2001, was referred to the urologist with hypogonadism and started TU in 2006 at age 59. Baseline $\mathrm{HbA}_{1 \mathrm{c}} 6.8 \%$, baseline HOMA-IR 8.4. From 27 months on TU onward, $\mathrm{HbA}_{1 \mathrm{c}}<6.5 \%$. Metformin discontinued after 7 years on TU. BMI reduced under testosterone from 31.3 to $25.5 \mathrm{~kg} / \mathrm{m}^{2}$, waist circumference from 96 to $86 \mathrm{~cm}$, weight loss: $18.4 \%$. Last obser

\#2: Patient with previous myocardial infarction; T2DM since 2002, received metformin since 2004, was referred by cardiologist because of sexual and urinary problems. Diagnosed with hypogonadism, started TU in 2006 at age 57. Baselin $\mathrm{HbA}_{1 \mathrm{c}} 7.2 \%$, baseline HOMA-IR 8.5. From 21 months on TU onward, $\mathrm{HbA}_{1 \mathrm{c}}<6.5 \%$. Metformin discontinued after 3 years on TU. BMI reduced under testosterone from 28.4 to $25.3 \mathrm{~kg} / \mathrm{m}^{2}$, waist circumference from 102 to $94 \mathrm{~cm}$, weight loss: $11 \%$. Last

\#3:T2DM since 2004, metformin since 2004, was referred by family physician because of sexual problems. Started TU in 2007 at age 60. Baseline $\mathrm{HbA}_{1 \mathrm{c}} 7.9 \%$, baseline HOMA-IR 2.6. From 45 months on $\mathrm{TU}$ onward, $\mathrm{HbA}_{1 \mathrm{c}}<6.5 \%$. Metformin discontinued after 2 years on T. BMI reduced under testosterone from 38.5 to $31.7 \mathrm{~kg} / \mathrm{m}^{2}$, waist circumference from 120 to $107 \mathrm{~cm}$, weight loss: $17.5 \%$. Last

\#4:T2DM since 2006, insulin since 2006, was referred by gastroenterologist with hypogonadism. Started TU in 2007 at age 56. Baseline $\mathrm{HbA}_{1 \mathrm{c}} 7.1 \%$, baseline HOMA-IR 2.9. From 57 months on $\mathrm{TU}$ onward, $\mathrm{HbA}_{1 \mathrm{C}}<6.5 \%$. Insulin discontinued after 3 years on TU. BMI reduced under testosterone from 25.4 to $24 \mathrm{~kg} / \mathrm{m}^{2}$, waist
circumference stable at $92 \mathrm{~cm}$, weight loss: $5.3 \%$. Last observation after 120 months on TU.
\#5:T2DM since 2003, metformin since 2003, insulin since 2005. Referred by family physician because of prostatitis and sexual problems. Gained $5 \mathrm{~kg}$ under From 87 months on TU onward, $\mathrm{HbA}_{1}<6.5 \%$. Insulin disco, From 87 months on TU onward, $\mathrm{HbA} A_{1 c}<6.5 \%$. Insulin discontinued after 7 , mettorwaist circumference from 110 to $98 \mathrm{~cm}$, weight loss: $21 \%$. Last observation after 117 months on TU.

\#6:T2DM since 2000, metformin since 2000, insulin since 2006. Referred by 政 insulin. Started TU in 2008 at age 68. Baseline $\mathrm{HbA}_{1 \mathrm{c}} 9.6 \%$, baseline HOMA-IR 12 insulin after 9 years TU onward, $\mathrm{HbA}_{1 \mathrm{c}}<6.5 \%$. Metformin discontinued after 5 , $\mathrm{kg} / \mathrm{m}^{2}$, waist circumference from 110 to $98 \mathrm{~cm}$, weight loss: $23.9 \%$. Last observation after 180 months on TU.

\#7:T2DM since 2006, metformin since 2006, insulin since 2007. Referred by family physician because of prostatitis and sexual problems. Gained $11 \mathrm{~kg}$ under insulin. Started TU in 2010 at age 52. Baseline $\mathrm{HbA}_{1 \mathrm{c}} 8.9 \%$, baseline HOMA-IR 9.4 . From 63 months on TU onward, $\mathrm{HbA}_{1<}<6.5 \%$. Metformin discontinued after 5 , $\mathrm{kg} / \mathrm{m}^{2}$, waist circumference from 113 to $103 \mathrm{~cm}$, weight loss: $18.5 \%$. Last observation after 85 months on TU.

\#8. Patient with CAD. T2DM since 2004, metformin since 2004. Referred by cardiologist because of erectile dysfunction. Started TU in 2010 at age 58 . Baseline HbA $7.2 \%$, baseline HOMA-IR 2.8. From 45 months on TU onward, $\mathrm{HbA}_{<}<6.5 \%$ Metformin discontinued after 3 years on TU. BMI reduced under testosterone from 35.2 to $29.1 \mathrm{~kg} / \mathrm{m}^{2}$, waist circumference from 107 to $96 \mathrm{~cm}$, weight loss: $17.4 \%$. Last observation after 75 months on TU.
$\mathrm{HbA}_{1}(\%)$ in 8 hypogonadal men with T2DM on long-term treatment with testosterone undecanoate

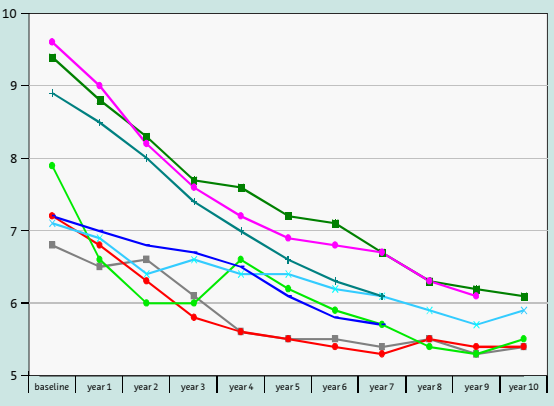

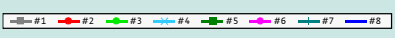

HOMA-IR in 8 hypogonadal men with T2DM on long-tem treatment with testosterone undecanoate

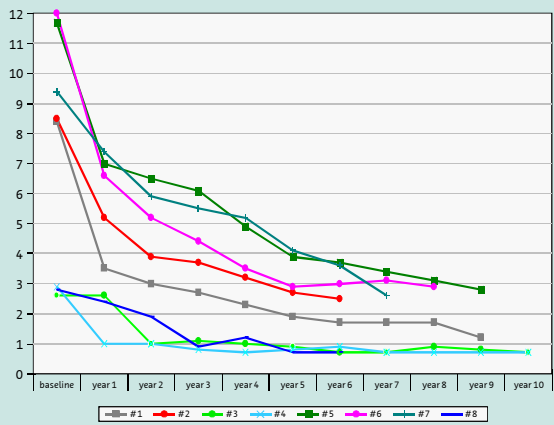

Insulin dose (U/d) in 4 hypogonadal men with T2DM on long-term treatment with testosterone undecanoate

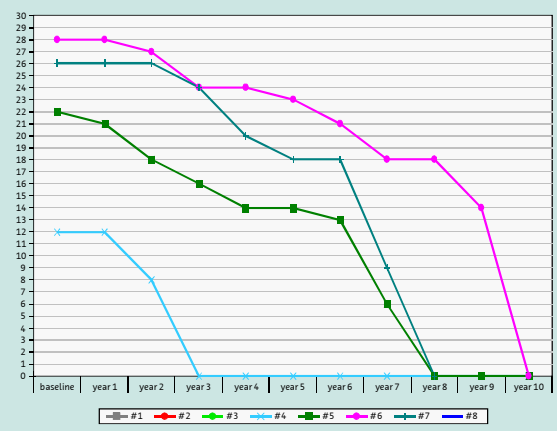

BMI $\left(\mathrm{kg} / \mathrm{m}^{2}\right)$ in 8 hypogonadal men with T2DM on long-term treatment with testosterone undecanoate

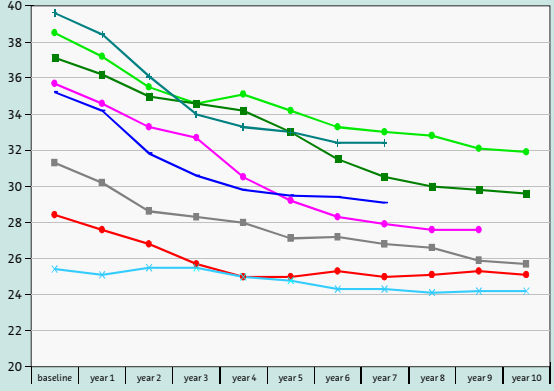



Waist circumference $(\mathrm{cm})$ in 8 hypogonadal men with T2DM on long-term treatment with testosterone undecanoate

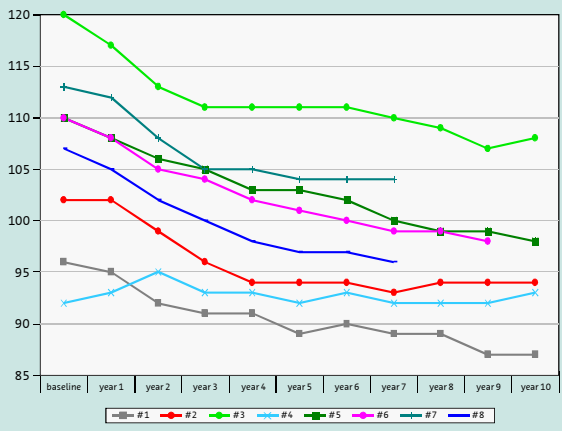

